\title{
FORMAL TORSORS UNDER REDUCTIVE GROUP SCHEMES
}

\author{
BENEDICTUS MARGAUX
}

\begin{abstract}
We consider the algebraization problem for torsors over a proper formal scheme under a reductive group scheme. Our results apply to the case of semisimple group schemes (which is addressed in detail).
\end{abstract}

\section{INTRODUCTION}

Throughout this paper $R$ will be a complete noetherian local ring with maximal ideal $\mathfrak{m}$. We put $R_{n}=R / \mathfrak{m}^{n+1}$ for each $n \geq 0$. The natural map $R \rightarrow \varliminf_{n} R_{n}$ is a ring isomorphism and we will henceforth identify these two rings.

For the theory of formal schemes over $R$, we refer the reader to [8, $\S 10],[12$, $\S$ II.9] and [16, Tag 0AHW, $\S 79]$ I $^{1}$ Let $X$ be a proper $R$-scheme, and let $\widehat{X}$ be the associated formal scheme. Grothendieck's existence theorem provides an equivalence of categories between the category of coherent sheaves over $X$ and the category of coherent sheaves on the formal scheme $\widehat{X}[9,5.1 .4]$, [13, §8.4]. The restriction to locally trivial coherent sheaves of constant rank $r$ yields a natural equivalence between the category of $\mathrm{GL}_{r}$-torsors over $X$ and the category of $\widehat{\mathrm{GL}}_{r}$-torsors over $\widehat{X}$.

The purpose of the paper is to extend this statement to a larger class of affine group schemes over $X$ which includes semisimple group schemes. This question has been also studied by Baranovsky [2, §3], but only for group schemes arising from $R$-group schemes by base change.

Conventions on vector groups and linear groups. We use mainly the terminology and notation of Grothendieck-Dieudonné [8, §9.4 and 9.6], which agrees with that of Demazure-Grothendieck used in [15, Exp. I.4]

Let $S$ be a scheme and let $\mathcal{E}$ be a quasi-coherent sheaf over $S$. For each morphism $f: T \rightarrow S$, we denote by $\mathcal{E}_{(T)}=f^{*}(\mathcal{E})$ the inverse image of $\mathcal{E}$ by the morphism $f$. Recall that the $S$-scheme $\mathbf{V}(\mathcal{E})=\operatorname{Spec}\left(\operatorname{Sym}^{\bullet}(\mathcal{E})\right)$ is affine over $S$ and represents the $S$-functor $T \mapsto \operatorname{Hom}_{\mathcal{O}_{T}}\left(\mathcal{E}_{(T)}, \mathcal{O}_{T}\right)$ [8, 9.4.9].

We assume now that $\mathcal{E}$ is locally free of finite rank and denote by $\mathcal{E}^{\vee}$ its dual. In this case the affine $S$-scheme $\mathbf{V}(\mathcal{E})$ is of finite presentation [8, 9.4.11]; also the

2010 Mathematics Subject Classification. 14D15, 14L30.

Key words and phrases. Torsors; Formal schemes; Reductive group schemes; Algebraization.

${ }^{1}$ Since the numbering of the Stacks Project [16] evolves over time, we also provide the relevant tags. 
$S$-functor $T \mapsto H^{0}\left(T, \mathcal{E}_{(T)}\right)=\operatorname{Hom}_{\mathcal{O}_{T}}\left(\mathcal{O}_{T}, \mathcal{E}_{(T)}\right)$ is representable by the affine $S$ scheme $\mathbf{V}\left(\mathcal{E}^{\vee}\right)$ which is also denoted by $\mathbf{W}(\mathcal{E})$ [15, I.4.6].

The above considerations apply to the locally free coherent sheaf $\mathcal{E} n d(\mathcal{E})=$ $\mathcal{E}^{\vee} \otimes_{\mathcal{O}_{S}} \mathcal{E}$ over $S$ so that we can consider the affine $S$-scheme $\mathbf{V}(\mathcal{E} n d(\mathcal{E}))$ which is an $S$-functor in associative commutative and unital algebras [8, 9.6.2]. Now we consider the $S$-functor $T \mapsto \operatorname{Aut}_{\mathcal{O}_{T}}\left(\mathcal{E}_{(T)}\right)$. It is representable by an open $S$ subscheme of $\mathbf{V}(\mathcal{E} n d(\mathcal{E}))$ which is denoted by $\operatorname{GL}(\mathcal{E})$ [8, 9.6.4].

We set $\mathrm{GL}_{r, S}=\operatorname{GL}\left(\mathcal{O}_{S}^{r}\right)$ for each $r \geq 1$. If $S=\operatorname{Spec}(A)$ is affine, then $\mathcal{E}=\mathcal{O}_{S}^{r}$ corresponds to the $A$-module $E=A^{r}$. In this case we will use the notation $\mathrm{GL}_{r}(E)$ instead of $\mathrm{GL}_{r, S}$. Finally, for scheme morphisms $Y \rightarrow X \rightarrow S$, we denote by $\prod_{X / S}(Y / X)$ the $S$-functor defined by

$$
\left(\prod_{X / S}(Y / X)\right)(T)=Y\left(X \times{ }_{S} T\right)
$$

for each $S$-scheme $T$. Recall that if $\prod_{X / S}(Y / X)$ is representable by an $S$-scheme, this scheme is called the Weil restriction of $Y$ to $S$.

\section{Formal TORSORS}

Let $R$ be as above, and let $X$ be a proper $R$-scheme. We start with the following key observation about limits.

Lemma 2.1. Let $f: Y \rightarrow X$ be a separated morphism of finite type. Then the natural map

$$
\left(\prod_{X / R}(Y / X)\right)(R) \rightarrow \underset{n}{\varliminf_{n}}\left(\prod_{X / R}(Y / X)\right)\left(R_{n}\right)=\underset{n}{\lim _{n}}\left(\prod_{X_{n} / R_{n}}\left(Y_{n} / X_{n}\right)\right)\left(R_{n}\right)
$$

is bijective.

Proof. The last equality follows from the fact that $\prod_{X / S}(Y / X)$ commutes with base change. Consider the commutative diagram

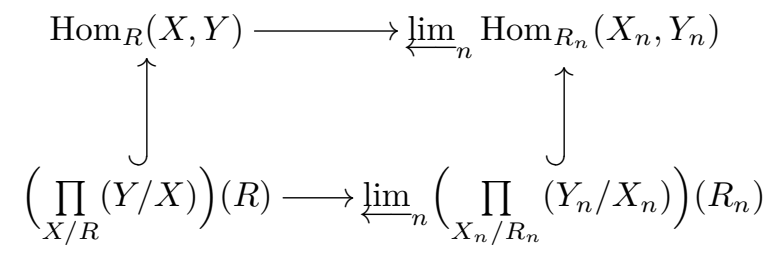

According to [16, Tag 0898, 29.28.3], the top horizontal map is bijective so that the bottom horizontal map is injective. Let $\left(s_{n}: X_{n} \rightarrow Y_{n}\right)_{n \geq 0}$ be a coherent family of sections. It lifts to a (unique) morphism $s: X \rightarrow Y$. Then the morphism $g=$ $f \circ s: X \rightarrow X$ is such that $g_{n}=i d_{X_{n}}$ for all $n \geq 0$. Since the map $\operatorname{Hom}_{R}(X, X) \rightarrow$ $\varliminf_{n} \operatorname{Hom}_{R_{n}}\left(X_{n}, X_{n}\right)$ is bijective, we conclude that $g=i d_{X}$ whence $s$ is a section $\stackrel{n}{Y} \rightarrow X$. We have shown the surjectivity of the bottom map. 
Let $\mathfrak{G}$ be an affine $X$-group scheme of finite presentation. We set $X_{n}=X \times{ }_{R} R_{n}$ and $\mathfrak{G}_{n}=\mathfrak{G} \times_{X} X_{n}$ for each $n \geq 0$. We denote by $\widehat{\mathfrak{G}}=\left(\mathfrak{G}_{n}\right)_{n \geq 0}$ the formal group scheme over $\widehat{X}$ attached to $\mathfrak{G}$.

A formal $\widehat{\mathfrak{G}}$-torsor $\widehat{\mathfrak{P}}$ is the data of a $\mathfrak{G}_{n}$-torsor $\mathfrak{P}_{n}$ over $X_{n}$ for each $n \geq 0$ together with compatible $\mathfrak{G}_{n+1}$-isomorphisms $\theta_{n}: \mathfrak{P}_{n+1} \times_{R_{n+1}} R_{n} \stackrel{\sim}{\longrightarrow} \mathfrak{P}_{n}$. If $\mathfrak{P}$ is a $\mathfrak{G}$-torsor, $\widehat{\mathfrak{P}}$ is a formal $\widehat{\mathfrak{G}}$-torsor and this assignment is faithful in the following sense.

Lemma 2.2. Let $\mathfrak{P}, \mathfrak{Q}$ be two $\mathfrak{G}$-torsors. The natural map $\operatorname{Isom}_{\mathfrak{G}}(\mathfrak{P}, \mathfrak{Q}) \rightarrow$ $\operatorname{Isom}_{\widehat{\mathfrak{G}}}(\widehat{\mathfrak{P}}, \widehat{\mathfrak{Q}})$ is bijective.

Proof. Up to replacing $\mathfrak{G}$ (resp. $\mathfrak{Q}$ ) by the twisted $R$-group scheme $\mathfrak{P} \mathfrak{G}$ (resp. $\left.\mathfrak{P}^{o p} \wedge{ }^{\mathfrak{G}} \mathfrak{Q}\right)$, we may assume that $\mathfrak{P}=\mathfrak{G}$. In this case, we have $\operatorname{Isom}_{\mathfrak{G}}(\mathfrak{P}, \mathfrak{Q})=$ $\mathfrak{Q}(X)$ so that our original question is reduced to showing that the natural map

$$
\mathfrak{Q}(X) \rightarrow \underbrace{\lim }_{n} \mathfrak{Q}_{n}\left(X_{n}\right)
$$

is bijective. Locally for the fppf topology, $\mathfrak{Q}$ is isomorphic to $\mathfrak{G}$. According to the permanence properties of faithfully flat descent $\mathfrak{Q}$ is affine of finite presentation over $X[10,2.7 .1$.(vi) and (xiii)]. So Lemma 2.1 applies and shows that the above map is bijective.

2.1. Algebraizable torsors. We say that a formal $\widehat{\mathfrak{G}}$-torsor $\widehat{\mathfrak{P}}$ is algebraizable if it arises from a $\mathfrak{G}$-torsor $\mathfrak{P}$. Lemma 2.2 shows that if such a $\mathfrak{P}$ exists, it is unique up to isomorphism.

Lemma 2.3. Let $\mathfrak{G}$ and $\mathfrak{G}^{\prime}$ be two $X$-group schemes which are affine and of finite presentation. Assume that $\mathfrak{G}$ is flat and that $i: \mathfrak{G} \rightarrow \mathfrak{G}^{\prime}$ is a monomorphism of $X$-group schemes with the property that the fppf quotient $\mathfrak{G}^{\prime} / \mathfrak{G}$ is representable by an affine $X$-scheme $\mathfrak{Q}$.

Let $\widehat{\mathfrak{F}}$ be a $\widehat{\mathfrak{G}}$-torsor and denote by $\widehat{\mathfrak{F}}^{\prime}=i_{*}(\widehat{\mathfrak{F}})$ the corresponding $\widehat{\mathfrak{G}}^{\prime}$-torsor. Then $\widehat{\mathfrak{F}}$ is algebraizable if and only if $\widehat{\mathfrak{F}}^{\prime}$ is algebraizable.

Proof. It is clear that if $\widehat{\mathfrak{F}}$ is algebraizable then so is $\widehat{\mathfrak{F}}^{\prime}$. Conversely, assume that the $\widehat{\mathfrak{G}}^{\prime}$-torsor $\widehat{\mathfrak{F}}^{\prime}$ is algebraizable, i.e. it arises from a $\mathfrak{G}^{\prime}$-torsor $\mathfrak{F}^{\prime}$. We consider the affine $X$-scheme $\mathfrak{Z}=\mathfrak{F}^{\prime} / \mathfrak{G}:=\mathfrak{F}^{\prime} \wedge^{\mathfrak{G}^{\prime}}\left(\mathfrak{G}^{\prime} / \mathfrak{G}\right)$; the reduction of $\mathfrak{F}^{\prime}$ to $\mathfrak{G}$ defined by faithfully flat descent. According to [15, $\mathrm{VI}_{B} \cdot 9.2$.(xiii).b], the $X$-scheme $\mathfrak{G}^{\prime} / \mathfrak{G}$ is of finite presentation. Since $\mathfrak{Z}$ is locally isomorphic to $\mathfrak{G}^{\prime} / \mathfrak{G}$ with respect to the fppf topology, the permanence properties of faithfully flat descent show that $\mathfrak{Z}$ is affine of finite presentation over $X[10,2.7 .1 .(v i)$ and (xiii)]. According to Lemma 2.1. the map $\mathfrak{Z}(X) \rightarrow \varliminf_{n} \mathfrak{Z}_{n}\left(X_{n}\right)$ is bijective.

Each $\mathfrak{F}_{n}$ defines a point $z_{n} \in \mathfrak{Z}\left(R_{n}\right)$ in a coherent way so that we get a point $z \in \mathfrak{Z}(R)$. That point defines a reduction of the $\mathfrak{G}^{\prime}$-torsor $\mathfrak{F}^{\prime}$ to a $\mathfrak{G}$-torsor $\mathfrak{F}[7$, III.3.2.1]. Since $z$ maps to $z_{n}$, we have $\mathfrak{F}_{R_{n}}=\mathfrak{F}_{n}$ for each $n \geq 0$. Thus $\widehat{\mathfrak{F}}$ is algebraizable. 


\section{Representations of GROUP schemes}

3.1. The Chevalley case. Let $G$ be a reductive split $\mathbb{Z}$-group scheme and we denote by $G_{a d}$ its adjoint quotient. We remind the reader that the functor of automorphisms of $G$ is representable by a smooth $\mathbb{Z}$-group scheme $\operatorname{Aut}(G)$ [15. XXIV.1]. Furthermore there is an exact sequence of $\mathbb{Z}$-group schemes

$$
1 \rightarrow G_{a d} \stackrel{\text { int }}{\rightarrow} \operatorname{Aut}(G) \stackrel{\pi}{\rightarrow} \operatorname{Out}(G) \rightarrow 1
$$

where $\operatorname{Out}(G)$ is a constant group scheme. In other words, Out $(G)$ is the $\mathbb{Z}$-group scheme attached to the abstract group $\operatorname{Out}(G)(\mathbb{Z})$. In the semisimple case $\operatorname{Out}(G)$ is finite (and in particular $\operatorname{Aut}(G)$ is affine). This is not the case in general. For example, Aut $\left(\mathbb{G}_{m}^{2}\right)$ is the constant $\mathbb{Z}$-group scheme attached to the abstract group $\mathrm{GL}_{2}(\mathbb{Z})$.

Let $\Gamma$ be a finite subgroup of $\operatorname{Out}(G)(\mathbb{Z})$. We get a monomorphism of $\mathbb{Z}$-group schemes $\Gamma_{\mathbb{Z}} \rightarrow \operatorname{Out}(G)$ and consider the $\mathbb{Z}$-group scheme

$$
\operatorname{Aut}_{\Gamma}(G)=\operatorname{Aut}(G) \times \operatorname{Out}(G) \Gamma_{\mathbb{Z}},
$$

obtained by pullback. The above yields the exact sequence

$$
1 \rightarrow G_{a d} \rightarrow \operatorname{Aut}_{\Gamma}(G) \stackrel{\pi}{\rightarrow} \Gamma_{\mathbb{Z}} \rightarrow 1 .
$$

Since $\Gamma_{\mathbb{Z}}$ and $G_{a d}$ are smooth affine over $\mathbb{Z}$, so is $\operatorname{Aut}_{\Gamma}(G)\left[15, \mathrm{VI}_{B}\right.$ 9.2.(viii)].

Lemma 3.1. There exists a free $\mathbb{Z}$-module of finite type $E$, and a closed immersion $\mathbb{Z}$-group scheme homomophism $i: G \rtimes \operatorname{Aut}_{\Gamma}(G) \rightarrow \mathrm{GL}(E)$ such that the fppf quotient sheaf $\mathrm{GL}(E) / G$ (resp. $\left.\mathrm{GL}(E) /\left(G \rtimes \operatorname{Aut}_{\Gamma}(G)\right), \mathrm{GL}(E) / G_{a d}\right)$ is representable by a smooth affine $\mathbb{Z}$-scheme.

Proof. Since $G \rtimes \operatorname{Aut}_{\Gamma}(G)$ is an affine smooth $\mathbb{Z}$-group scheme, there exists a free $\mathbb{Z}$-module of finite rank $E$ and a faithful linear representation $\rho: G \rtimes \operatorname{Aut}_{\Gamma}(G) \rightarrow$ $\mathrm{GL}(E)$ which is a closed immersion [3, 1.4.5].

The fppf sheaf $\operatorname{GL}(E) /\left(G \rtimes \operatorname{Aut}_{\Gamma}(G)\right)$ is representable by a $\mathbb{Z}$-scheme [1, Th. IV.4.B] which is smooth and separated [15, $\mathrm{VI}_{B} \cdot 9.2 .(\mathrm{x})$ and (xii)]. The $\mathbb{Z}$-group scheme $G \rtimes G_{a d}$ is reductive. According to [4, 6.12.ii], the fppf sheaf $\mathrm{GL}(E) /\left(G \rtimes G_{a d}\right)$ is representable by an affine smooth $\mathbb{Z}$-scheme and so are $\mathrm{GL}(E) / G$ and $\mathrm{GL}(E) / G_{a d}$. Since the map $\operatorname{GL}(E) /\left(G \rtimes G_{a d}\right) \rightarrow \mathrm{GL}(E) /\left(G \rtimes \operatorname{Aut}_{\Gamma}(G)\right)$ is a $\Gamma_{\mathbb{Z}}$-torsor, it is a finite étale cover. It follows that $\operatorname{GL}(E) /\left(G \rtimes \operatorname{Aut}_{\Gamma}(G)\right)$ is affine [16, Tag 01YN, Lemma 29.13.3]. Similarly the $\mathbb{Z}$-scheme $\operatorname{GL}(E) / \operatorname{Aut}_{\Gamma}(G)$ is affine.

3.2. An isotriviality condition. In this section, we assume that the base scheme $S$ is noetherian and we are given a reductive $S$-group scheme $\mathfrak{G}$ of constant type. Thus, there exists a Chevalley $\mathbb{Z}$-group scheme $G$ such that $\mathfrak{G}$ is locally isomorphic to $G_{S}$ for the étale topology [15, XXII.2.3, 2.5]. Also the fppf $\operatorname{sheaf} \underline{\operatorname{Isom}}\left(G_{S}, \mathfrak{G}\right)$ is representable by a $\operatorname{Aut}(G)_{S}$-torsor $\operatorname{Isom}\left(G_{S}, \mathfrak{G}\right)$ defined in [15, XXIV.1.8]. The contracted product $\operatorname{Isomext}\left(G_{S}, \mathfrak{G}\right):=\operatorname{Isom}\left(G_{S}, \mathfrak{G}\right) \wedge \operatorname{Aut}(G)_{S} \operatorname{Out}(G)_{S}$ is a $\operatorname{Out}(G)_{S^{-}}$ torsor [15, XXIV.1.10] which encodes the isomorphism class of the quasi-split form of $\mathfrak{G}$. 
Proposition 3.2. We assume that the $\operatorname{Out}(G)_{S}$-torsor $\operatorname{Isomext}\left(G_{S}, \mathfrak{G}\right)$ is isotrivial, i.e. there exists a finite étale cover $S^{\prime} / S$ such that $\operatorname{Isomext}\left(G_{S}, \mathfrak{G}\right)\left(S^{\prime}\right) \neq \emptyset$. Then there exists a locally free coherent $\mathcal{O}_{S}$-module $\mathcal{E}$, and a closed immersion $S$-group scheme homomorphism $i: \mathfrak{G} \rightarrow \mathrm{GL}(\mathcal{E})$ such that the fppf quotient sheaf $\mathrm{GL}(\mathcal{E}) / \mathfrak{G}$ is representable by a smooth affine $S$-scheme.

Remark 3.3. (a) If $G$ is semisimple, $\operatorname{Out}(G)$ is a finite constant group so that the isotriviality condition is obviously satisfied.

(b) If $S$ is a normal connected scheme, the isotriviality condition is satisfied since Isomext $\left(G_{S}, \mathfrak{G}\right) \rightarrow S$ is a $\operatorname{Out}(G)_{S}$-cover [15, X.6.2 and 5.14].

Proof. The noetherian assumption reduces the problem to the connected case (in particular, $S$ is non-empty by convention [16, Tag 004R, 5.7.1]). We consider the $\operatorname{Aut}(G)_{S}$-torsor $\mathfrak{E}=\operatorname{Isom}\left(G_{S}, \mathfrak{G}\right)$ defined above; we have $\mathfrak{G}={ }^{\mathfrak{E}}\left(G_{S}\right)$, i.e. $\mathfrak{G}$ is the twist of $G_{S}$ by the $\operatorname{Aut}(G)_{S}$-torsor $\mathfrak{E}$.

The isotriviality assumption for the $\operatorname{Out}(G)_{S}$-torsor $\mathfrak{F}=\mathfrak{E} \wedge \operatorname{Aut}(G)_{S} \operatorname{Out}(G)_{S}$ means that there exists a finite étale cover $S^{\prime} / S$ such that $\mathfrak{F}\left(S^{\prime}\right) \neq \emptyset$. Grothendieck's theory of the algebraic fundamental group [14] permits to assume that $S^{\prime}$ is connected and that $S^{\prime} \rightarrow S$ is a $\Theta_{S^{-}}$-torsor, where $\Theta$ is a finite abstract group.

We have a bijection $H^{1}\left(\Theta, \operatorname{Out}(G)\left(S^{\prime}\right)\right) \stackrel{\sim}{\longrightarrow} H^{1}\left(S^{\prime} / S\right.$, Out $\left.(G)\right)$ [6] end of $\left.\S 2.2\right]$. Since $S^{\prime}$ is connected, we have $\operatorname{Out}(G)(\mathbb{Z})=\operatorname{Out}(G)\left(S^{\prime}\right)$ so that the action of $\Theta$ on $\operatorname{Out}(G)\left(S^{\prime}\right)$ is trivial. We have then a bijection

$$
\operatorname{Hom}_{g r}(\Theta, \operatorname{Out}(G)(\mathbb{Z})) / \operatorname{Out}(G)(\mathbb{Z}) \stackrel{\sim}{\longrightarrow} H^{1}\left(\Theta, \operatorname{Out}(G)\left(S^{\prime}\right)\right) .
$$

It follows that the class of the $\operatorname{Out}(G)_{S}$-torsor $\mathfrak{F}$ is given by the conjugacy class of a homomorphism $\rho: \Theta \rightarrow \operatorname{Out}(G)(\mathbb{Z})$.

Let $\Gamma=\operatorname{Im}(\rho)$, it is a finite subgroup of $\operatorname{Out}(G)(\mathbb{Z})$. We consider the $\mathbb{Z}$ group scheme $\operatorname{Aut}_{\Gamma}(G)=\pi^{-1}(\Gamma)$ as in the previous section. The isomorphism $\operatorname{Aut}(G)_{S} / \operatorname{Aut}_{\Gamma}(G)_{S} \stackrel{\sim}{\longrightarrow} \operatorname{Out}(G)_{S} / \Gamma_{S}$ induces an isomorphism $\mathfrak{E} / \operatorname{Aut}_{\Gamma}(G)_{S} \stackrel{\sim}{\longrightarrow}$ $\mathfrak{F} / \Gamma_{S}$. The reduction of the $\operatorname{Out}(G)_{S}$-torsor $\mathfrak{F}$ to $\Gamma_{S}$ defines then a reduction of the $\operatorname{Aut}(G)_{S}$-torsor $\mathfrak{E}$ to a $\operatorname{Aut}_{\Gamma}(G)_{S}$-torsor $\mathfrak{E}_{\sharp}$ [7, III.3.2.1].

Remark 3.4. (a) If $G$ is semisimple, we can take in the proof $\Gamma=\operatorname{Out}(G)(\mathbb{Z})$. We thus find a $\mathcal{O}_{S}$-coherent sheaf $\mathcal{E}$ as desired which is $\mathfrak{G} \rtimes \operatorname{Aut}(\mathfrak{G})$-equivariant.

(b) Thomason has proven stronger statements than Proposition 3.2 for embedding group schemes in linear group schemes [17, §3].

\section{Main Statement}

The following generalization of Grothendieck's existence theorem strengthens Baranovsky's result [2, Th. 3.1].

Theorem 4.1. Let $R$ be a complete noetherian local ring. Let $X$ be a proper $R$-scheme and let $\widehat{X}$ be the associated formal scheme. Let $G$ be a Chevalley $\mathbb{Z}$ group scheme and let $\mathfrak{G}$ be an $X$-form of $G_{X}$. Assume that the Out $(G)_{X}$-torsor Isomext $\left(G_{X}, \mathfrak{G}\right)$ is isotrivial. Then, 
(1) The assignment $\mathfrak{P} \mapsto \widehat{\mathfrak{P}}$ induces an equivalence of categories between the category of $\mathfrak{G}$-torsors of $X$ and that of $\widehat{\mathfrak{G}}$-torsors over $\widehat{X}$.

(2) Assume that $\mathfrak{G}$ is semisimple. For $\mathfrak{H}=\mathfrak{G}$, Aut $(\mathfrak{G}), \mathfrak{G} \rtimes \operatorname{Aut}(\mathfrak{G})$ the assignment $\mathfrak{P} \mapsto \widehat{\mathfrak{P}}$ induces an equivalence of categories between the category of $\mathfrak{H}$-torsors of $X$ and that of $\widehat{\mathfrak{H}}$-torsors over $\widehat{X}$.

Proof. (1) By Lemma 2.2 we have only to show algebraization. The $R$-scheme $X$ is proper, namely separated, of finite type, and universally closed. Since $R$ is noetherian, $X$ is locally noetherian. Also the morphism $X \rightarrow \operatorname{Spec}(R)$ is quasicompact [16, Tag 04XU, 28.39.9] so that $X$ is quasi-compact. The scheme $X$ is quasi-compact and locally noetherian, hence is noetherian by definition [16, Tag 01OU, 27.5.1]. Without loss of generality we may assume that $X$ is connected.

Proposition 3.2 provides a closed immersion $i: \mathfrak{G} \rightarrow \operatorname{GL}(\mathcal{E})$ where $\mathcal{E}$ is a locally free coherent $\mathcal{O}_{X}$-module and such that the fppf quotient sheaf $\mathrm{GL}(\mathcal{E}) / \mathfrak{G}$ is representable by a smooth affine $X$-scheme. Lemma 2.3 reduces the algebraization problem to the case of $\operatorname{GL}(\mathcal{E})$. Since $X$ is connected, $\mathcal{E}$ is locally free of rank $r$. We consider the $\mathrm{GL}_{r}$-torsor $\mathfrak{Q}=\operatorname{Isom}\left(\mathcal{O}_{X}^{r}, \mathcal{E}\right)$ over $X$. Torsion by $\mathfrak{Q}$ (resp. $\widehat{\mathfrak{Q}}$ ) induces an equivalence of categories between the category of $\mathrm{GL}_{r}$-torsors (resp. $\widehat{\mathrm{GL}}_{r}$-torsors) and that of $\mathrm{GL}(\mathcal{E})$-torsors (resp. $\widehat{\mathrm{GL}(\mathcal{E})}$-torsors). It follows that the algebraization question is equivalent for $\mathrm{GL}_{r}$-torsors and for $\mathrm{GL}(\mathcal{E})$-torsors. Grothendieck's existence theorem states that $\mathrm{GL}_{r}$-torsors over $\widehat{X}$ are algebraizable. Thus algebraization holds for $\operatorname{GL}(\mathcal{E})$ and for $\mathfrak{G}$.

(2) Remark 3.4 . (a) shows that the representation $\mathfrak{G} \rightarrow \mathrm{GL}(\mathcal{E})$ arises from a representation $\mathfrak{G} \rtimes \operatorname{Aut}(\mathfrak{G}) \rightarrow \operatorname{GL}(\mathcal{E})$. The same argument applies then to $\mathfrak{G} \rtimes \operatorname{Aut}(\mathfrak{G})$ and $\operatorname{Aut}(\mathfrak{G})$.

4.1. Examples and applications. Let $d \geq 1$ be a positive integer. If we consider the case of $\mathfrak{G}=\mathrm{PGL}_{n}$ and use the dictionary given in [11, §7] between $\mathrm{PGL}_{d^{-}}$ torsors and Azumaya algebras of degree $d$, we get an algebraization statement for Azumaya algebras of degree $d$.

Corollary 4.2. There is an equivalence of categories between Azumaya algebras over $X$ (of degree d) and formal degree $d$ Azumaya algebras over $\widehat{X}$ (of degree $d$ ).

Similarly, by considering the case of the Chevalley $\mathbb{Z}$-group scheme of type $G_{2}$, we obtain an equivalence of categories octonion algebras over $X$ and formal octonion algebras over $\widehat{X}$ [5, App. B].

More generally for the group scheme $\operatorname{Aut}(G)$ of a semisimple Chevalley $\mathbb{Z}$-group $G$ we have the following fact as a special case of Theorem 4.1.(2).

Corollary 4.3. There is an equivalence of categories between the groupoid of $X$ forms of $G_{X}$ and that of formal $\widehat{X}$-forms of $\widehat{G}_{X}$.

In particular, we obtain the following fact. 
Corollary 4.4. Assume that we are given a formal $\widehat{X}$-group scheme $\widehat{\mathfrak{G}}$ such that each $\mathfrak{G}_{n}$ is an $X_{n}$-form of $G_{X_{n}}$. Then $\widehat{\mathfrak{G}}$ is algebraizable in a semisimple $X$-group scheme $\mathfrak{G}$ which is a $X$-form of $G_{X}$.

\section{ACKNOWLEDGEMENT}

My sincere gratitude to the referee for his/her careful reading of the original version, and the valuable corrections and feedback.

\section{REFERENCES}

[1] S. Anantharaman, Schémas en groupes, espaces homogènes et espaces algébriques sur une base de dimension 1, Sur les groupes algébriques, pp. 5-79. Bull. Soc. Math. France, Mém. 33, Soc. Math. France, Paris, 1973. MR 0335524.

[2] V. Baranovsky, Algebraization of bundles on non-proper schemes, Trans. Amer. Math. Soc. 362 (2010), no. 1, 427-439. MR 2550158

[3] F. Bruhat, J. Tits, Groupes réductifs sur un corps local : II. Schémas en groupes. Existence d'une donnée radicielle valuée, Publications Mathématiques de l'I.H.É.S. 60 (1984), 5-184. MR 0756316

[4] J.-L. Colliot-Thélène, J.-J. Sansuc, Fibrés quadratiques et composantes connexes réelles, Math. Annalen 244 (1979), no. 2, 105-134. MR 0550842

[5] B. Conrad, Non-split reductive groups over Z, Autour des schémas en groupes. Vol. II, 193253, Panor. Synthèses, 46, Soc. Math. France, Paris, 2015. MR 3525597

[6] P. Gille, Sur la classification des schémas en groupes semi-simples, Autour des schémas en groupes. Vol. III, 39-110, Panor. Synthèses, 47, Soc. Math. France, Paris, 2015. MR 3525601.

[7] J. Giraud, Cohomologie non abélienne, Grundlehren der mathematischen Wissenschaften, 179, Springer, 1971. MR 0344253

[8] A. Grothendieck, J. A. Dieudonné, Eléments de géométrie algébrique. I, Grundlehren der Mathematischen Wissenschaften 166, Springer-Verlag, Berlin, 1971. MR 3075000.

[9] A. Grothendieck (avec la collaboration de J. Dieudonné), Eléments de Géométrie Algébrique II, Publications mathématiques de l'I.H.É.S. no. 11 and 17 (1961-1963).

[10] A. Grothendieck (avec la collaboration de J. Dieudonné), Eléments de Géométrie Algébrique $I V$, Publications mathématiques de l'I.H.É.S. no. 20, 24, 28 and 32 (1964-1967).

[11] A. Grothendieck, Le groupe de Brauer I : Algèbres d'Azumaya et interprétations diverses, Dix Exposés sur la Cohomologie des Schémas, 46-66, Adv. Stud. Pure Math., 3, NorthHolland, Amsterdam, 1968. MR 0244269

[12] R. Hartshorne, Algebraic Geometry, Graduate Texts in Mathematics, 52, Springer, 1977. MR 0463157

[13] L. Illusie, Grothendieck's existence theorem in formal geometry, with a letter of Jean-Pierre Serre, Math. Surveys Monogr., 123, Fundamental algebraic geometry, 179-233, Amer. Math. Soc., Providence, RI, 2005. MR 2223409.

[14] Revêtements étales et groupe fondamental. Séminaire de Géométrie Algébrique du Bois Marie 1960-1961 (SGA 1). Dirigé par Alexandre Grothendieck. Lecture Notes in Mathematics, 224. Springer-Verlag, Berlin-New York, 1971. MR 0354651. 
[15] Schémas en groupes. Séminaire de Géométrie Algébrique du Bois Marie 1962/64 (SGA 3). Dirigé par M. Demazure et A. Grothendieck. Lecture Notes in Mathematics, 151-153. Springer-Verlag, Berlin-New York, 1970. MR 0274458, MR 0274459, MR 0274460

[16] The Stacks Project, https://stacks.math.columbia.edu

[17] R. W. Thomason, Equivariant resolution, linearization, and Hilbert's fourteenth problem over arbitrary base schemes, Adv. in Math. 65 (1987), no. 1, 16-34. MR 0893468

\section{B. Margaux}

Laboratoire de Recherche "Princesse Stéphanie", Monte Carlo 51840, Monaco

benedictus.margaux@gmail.com

Received: May 29, 2018

Accepted: November 16, 2018 